OPEN ACCESS

Edited by:

Shuyu Zhang,

Sichuan University, China

Reviewed by:

Xi Yang,

Fudan University, China

Enrique Hernandez-Lemus, Instituto Nacional de Medicina Genómica (INMEGEN), Mexico

${ }^{*}$ Correspondence: Jinchang Wu wjinchang@sina.com Jundong Zhou zhoujundong330@163.com

${ }^{\dagger}$ These authors have contributed equally to this work

Specialty section: This article was submitted to Molecular and Cellular Oncology, a section of the journal Frontiers in Oncology

Received: 23 March 2021 Accepted: 15 June 2021 Published: 29 July 2021

Citation:

Li P, Xia X, Zhou J and Wu J (2021) Exploring the Pharmacological Mechanism of Radix Salvia Miltiorrhizae in the Treatment of Radiation Pneumonia by Using Network Pharmacology.

Front. Oncol. 11:684315 doi: 10.3389/fonc.2021.684315

\section{Exploring the Pharmacological Mechanism of Radix Salvia Miltiorrhizae in the Treatment of Radiation Pneumonia by Using Network Pharmacology}

\author{
Peng $\mathrm{Li}^{1+}$, Xiaochun $\mathrm{Xia}^{2+}$, Jundong Zhou ${ }^{3,4^{*}}$ and Jinchang $\mathrm{Wu}^{3,5^{*}}$
}

\begin{abstract}
${ }^{1}$ Department of Radiation Oncology, Huai'an Tumor Hospital \& Huai'an Hospital of Huai'an City, Huai'an, China, 2 Department of Radiation Oncology, Nantong Tumor Hospital, Affiliated Tumor Hospital of Nantong University, Nantong, China, ${ }^{3}$ Department of Radiation Oncology, Nanjing Medical University Affiliated Suzhou Hospital, Suzhou, China, ${ }^{4}$ Suzhou Cancer Center Core Laboratory, Nanjing Medical University Affiliated Suzhou Hospital, Suzhou, China, ${ }^{5}$ Department of Radiation Oncology, The Second Affiliated Hospital of Xuzhou Medical University, Xuzhou, China
\end{abstract}

Background: Radiation pneumonia $(\mathrm{RP})$ is the most common complication of radiotherapy to the thorax and seriously affects the survival rate and quality of life of patients. Radix Salviae Miltiorrhizae (RSM) is an ancient Chinese medicine, whose main pharmacological effect is to promote blood circulation and remove stasis. A growing number of studies have proved that RSM has a good effect on RP. However, the underlying mechanism is still unclear and needs to be fully elucidated.

Methods: The effective components and predictive targets of RSM were analyzed by Traditional Chinese Medicine Systems Pharmacology (TCMSP) database, and the related targets of RP were predicted by GeneCards database. The common targets of the two targets mentioned above were analyzed by protein-protein interaction on the STRING website, GO and KEGG analysis on the DAVID website, visualization by CytoScape3.7.0, and screening for Hubber gene by cytoHubber plug-in.

Results: A search of the TCMSP database revealed that RSM contains 65 chemical constituents and 165 potential protein targets. A total of 2,162 protein targets were found to be associated with RP. The top 10 hub genes were obtained by MCC algorithm for 70 common genes, including TP53, CASP3, MAPK1, JUN, VEGFA, STAT3, PTGS2, IL6, AKT1, and FOS. By analyzing the Gene Ontology, The anti-radiation pneumonia effect of $\mathrm{RSM}$ is that it performs molecular functions (protein homodimerization activity) in the nucleus through three biological processes (positive regulation of transcription from RNA polymerase II promoter,Extrinsic apoptotic signaling pathway in absence of ligand and lipopolysaccharide-mediated signaling pathway). Through KEGG analysis, the mechanism of RSM treatment of radiation pneumonia may be through PI3K-Akt, HIF-1, TNF signaling pathways. 


\section{Conclusions: Through network pharmacology analysis, we found the possible target genes of RSM on RP and revealed the most likely signaling pathway, providing theoretical basis for further elucidating the potential mechanism of RSM on RP.}

\section{Keywords: Radix Salviae Miltiorrhizae, radiation pneumonia, network pharmacology, thoracic neoplasms,} traditional Chinese medicine

\section{INTRODUCTION}

Radiation therapy (RT) has become one of the main treatments for thorax malignancies (1). The lung is a radiation-sensitive organ, so radiation pneumonia (RP) is the most common complication of chest radiotherapy, which limits the dose of tumor target and affects the effect of RT (2). With the advancement of modern radiotherapy equipment and technology (including IGRT, proton, and heavy particle therapy), the survival rate of radiotherapy for thorax tumors continues to increase, and the survival time is prolonged, but the proportion of patients with RP cannot be eliminated and increased year by year. The incidence of asymptomatic radiation pneumonia diagnosed by imaging is as high as $43 \%$, while the incidence of clinically diagnosed symptomatic radiation pneumonia is slightly lower at 5-15\% (3). Treatment and prevention of radiation pneumonia are urgently needed. As the exact mechanism of radiation pneumonia is still unclear, high-dose steroid hormone therapy is the main treatment of choice, and serious side effects such as immunosuppression and osteoporosis caused by high-dose hormones have limited their application, and patients treated with corticosteroids alone often relapse. In recent years, many traditional Chinese medicines for Blood Activating Stasis Removing Drugs have obtained good curative effect and research progress in the prevention and treatment of RP (4).

Radix Salvia Miltiorrhizae (RSM, Danshen in Chinese) is one of the oldest traditional Chinese medicines in China, with a history of more than 1,000 years of clinical application $(5,6)$. RSM has been included in the Chinese Pharmacopoeia since 1963. It is a common blood rheological agent that promotes blood circulation, stops bleeding and stasis, and nourishes and calms the nerves. Modern pharmacological research shows that RSM has the ability to protect vascular endothelial cells, prevent arrhythmia, prevent atherosclerosis, improve microcirculation, protect the heart muscle, inhibit and release platelet aggregation, increase coronary flow, increase the body's ability to resist hypoxia, and inhibit collagen fibers. It produces and promotes the degradation of fibrin, is anti-inflammatory, promotes antilipid peroxidation and scavenging free radicals, as well as protects liver cells and prevents pulmonary fibrosis (7-9). A growing number of studies have pointed out that RSM has a good effect on RP (10-15), but its underlying molecular mechanism is still unclear.

Network pharmacology came into being with the development of genomics, proteomics, and systems biology (16), by mining data on drugs and diseases in public databases, analyzing the active ingredients of drugs and related target genes of diseases, and studying the possible mechanism of action of drugs. Combining modern network pharmacology with traditional Chinese medicine is conducive to revealing the pharmacological effects of Chinese medicine from a complex network of traditional Chinese medicine with multiple components, multiple pathways, and multiple targets (17). In this study, the active ingredients of RSM were screened through the network pharmacology research method, and multiple targets and pathways for the treatment of radiation pneumonia were excavated to provide a reference for elucidating the mechanism of action of RSM.

\section{MATERIALS AND METHODS}

The main research process of this research is shown in the flowchart (Figure 1). First, we searched the active ingredients and putative target of RSM, and at the same time, we searched for the genes related to radiation pneumonia. The intersection of the above genes is used as the common genes for subsequent research. The protein-protein interaction network, hub gene, GO enrichment analysis, and KEGG analysis were analyzed separately.

\section{RSM Chemical Ingredients and Putative Target}

We used the Traditional Chinese Medicine System Pharmacology Database (18) (TCMSP ${ }^{\mathrm{TM}}$, http://tcmspw.com/ tcmsp.php) to retrieve the effective chemical ingredients of RSM and their putative targets. TCMSP is a systematic pharmacological platform for the study of herbal medicines. Its functions include the identification of the chemical constituents of herbal medicines and their corresponding targets. Absorption, distribution, metabolism, and excretion (ADME) are key processes that need to be considered in screening compounds for TCM. Oral bioavailability (OB) and drug similarity (DL) were the most important pharmacokinetic parameters. OB is the dose and rate at which the active ingredient of a drug enters the human bloodstream by mouth, and DL is used to evaluate the structural similarity between a drug and clinical treatment drugs in the drugbank database. The higher the $\mathrm{OB}$ and DL, the more likely it is to be an active ingredient in the drug. On the website, we searched for effective compounds by inputting Chinese pinyin "Danshen" and screened out effective compounds according to the criteria of oral bioavailability (OB) $>30 \%$ and drug likeness $(\mathrm{DL})>0.18$, and we found their corresponding target genes. 


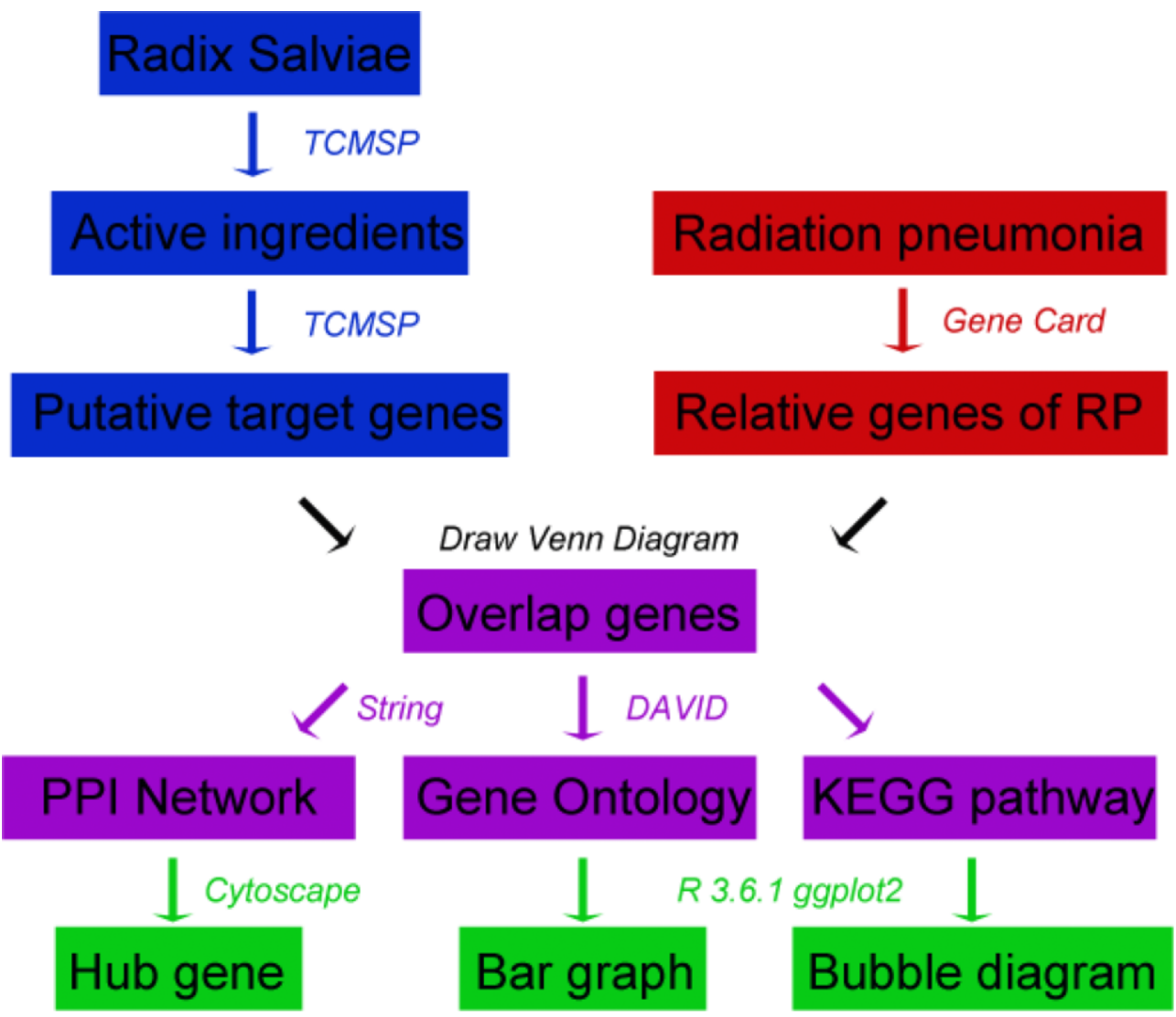

FIGURE 1 | Flowchart of the main research process.

\section{Genes Related to Radiation Pneumonia}

We searched genes related to radiation pneumonia through the Genecards website (GeneCards, https://www.genecards.org/) (19), which is an integrative database that includes genomic, transcriptomic, proteomic, genetic, clinical, and functional information. The current version is 4.12 and includes 13,878 disease genes. Using "radiation pneumonia" as the keyword, we established the disease target database of RP.

\section{Intersection of Drug Target Genes and Disease Target Genes}

We used Bioinformatics \& Evolutionary Genomics website (http://bioinformatics.psb.ugent.be/webtools/Venn/) to intersect the targets that match the active ingredients of RSM and the targets related to radiation pneumonia. The obtained overlapping genes are the key genes of RSM to act on radiation pneumonia.

\section{Traditional Chinese Medicine, Active Ingredient, Target Genes, Disease Network}

We inputted the abovementioned network data on traditional Chinese medicine, active ingredients of traditional Chinese medicine, target genes, and diseases into the software Cytoscape (http://cytoscape.org/, version 3.7.0) to obtain the topological network between them. Cytoscape is an open software platform for visualizing networks of biological pathways and molecular interaction networks, and it has rich plug-in functions.

\section{Protein-Protein Interaction Network and Hub Genes}

We used the String website and Cytoscape 3.7.1 software to analyze the key genes and build the network. The String database contains a large number of known or predicted PPI relationships. Cytoscape is a graph-oriented software for the analysis and visualization of genomic networks widely used in network pharmacology research. We uploaded the intersection gene to the String website (http://stringdb.org/, 10th edition) (20), with the restriction that the species selects "Homo sapiens" and the confidence is $>0.4$. The obtained protein-protein interaction network data were inputted into the software Cytoscape 3.7.0, and the Hub gene was obtained using the plug-in cytoHubber.

\section{Gene Ontology and KEGG Enrichment Analysis}

We performed Gene Ontology (GO) enrichment analysis and KEGG signal pathway enrichment analysis of intersection genes 
through the Database for Annotation, Visualization and Integrated Discovery (DAVID, https://david.ncifcrf.gov/home. jsp, ver. 6.8) (21, 22). GO enrichment mainly analyses the biological process, cellular composition, and molecular function of the target, whereas KEGG (www.kegg.jp/kegg/kegg1.html) enrichment analyzes the potential biological pathways and functions associated with the target. The data were visualized by software R language 3.6.1 and GGplot2 package.

\section{RESULTS}

\section{RSM Chemical Ingredients and Putative Targets}

We have screened 65 active compounds through the website according to the foregoing criteria. The detailed information are shown in Table 1, and 165 corresponding targets are shown in Table S1. Seven of the 65 compounds obtained had no corresponding target genes, and the final 58 compounds produced 165 putative targets.

\section{Genes Related to Radiation Pneumonia}

We used the key word "radiation pneumonia" and screened more than 2,000 genes related to radiation pneumonia through the website (see Table $\mathbf{S} 2$ for details).

\section{Traditional Chinese Medicine, Active Ingredient, Target Genes, Disease Network}

Through the software cytoscape3.7.0, a topological network between drug, chemical components, targets, and disease is constructed, as shown in Figure 2. The red octagon represents radiation pneumonia, the orange ovals represent the putative targets, the blue triangle represents RSM, and the pale blue rectangles represent the active ingredients. RSM has 58 active ingredients (seven active ingredients are not related to radiation pneumonia) and 70 targets related to radiation pneumonia. There are 358 links between 58 active ingredients and 70 target genes, as shown in Table S3.

\section{Intersection of Drug Target Genes and Disease Target Genes}

In order to clarify the pharmacological role of RSM in RP, as shown in Figure 3 of Venn, we matched the 165 genes predicted by RSM and the 2,162 genes predicted by RP to obtain 70 overlapping genes, which are probably the most critical genes for RSM to act on RP.

\section{Protein-Protein Interaction Network and Hub Genes}

The string website (STRING, https://string-db.org/) was used to build a network of 70 common genes, with 70 nodes and 903 edges (Figure 4A). By importing the above data into cytoscape3.7.0, and using the CytoHubber plug-in, the MCC algorithm yielded the top 10 hub genes (Figure 4B): TP53, CASP3, MAPK1, JUN, VEGFA, STAT3, PTGS2, IL6, AKT1, and FOS. The top 20 key genes obtained through the Degree algorithm are (Figure 4C) AKT1, TP53, EGFR, IL6, VEGFA, MYC, MAPK1, CASP3, JUN, STAT3, PTGS2, FOS, CCND1, TNF, ERBB2, BCL2L1, MMP9, MAPK14, AR, and CASP9. The above analysis suggests that these genes may be the key genes for RSM to act in RP.

\section{Gene Ontology and KEGG Enrichment Analysis}

We uploaded 70 common genes of RSM and RP to DAVID's website for GO analysis and KEGG analysis. Enrichment of Gene Oncology (molecular function $[\mathrm{MF}]$, biological process [BP], cellular components [CC]) is displayed in Figure 5. The p-values of these GO terms were less than 0.05, including protein homodimerization activity (MF), positive regulation of transcription from RNA polymerase II promoter (BP), extrinsic apoptotic signaling pathway in the absence of ligand (BP) and lipopolysaccharide-mediated signaling pathway (BP), and nucleus (CC).

Additionally, the results of the KEGG Pathway analysis showed that 70 genes were mapped in 50 signal pathways with significant differences $(\mathrm{P}<0.05)$, and the top 15 pathways are shown in Figure 6. Among them, 23, 15, and 15 genes were enriched in PI3K/AKT (EGFR, IL4, IL6, MCL1, RELA, MET, TP53, ITGB3, BCL2L1, AKT1, MAPK1, CDKN1A, CCND1, CASP9, GSK3B, BCL2, VEGFA, PIK3CA, MDM2, NOS3, INSR, MYC, IL2), HIF-1 (EGFR, IL6, RELA, ERBB2, EDN1, STAT3, AKT1, MAPK1, CDKN1A, BCL2, VEGFA, IFNG, PIK3CA, NOS3, INSR), and TNF (ICAM1, IL6, TNF, PTGS2, RELA, MMP9, EDN1, NFKBIA, AKT1, FOS, MAPK1, CASP3, MAPK14, JUN, PIK3CA) pathway, respectively. The diagram of the above signaling pathways is shown in Figure 7 . These pathways are closely related to cell proliferation, differentiation, apoptosis, and adhesion, so we believe that RSM may improve the occurrence and development of radiation pneumonia through these pathways.

\section{DISCUSSION}

$\mathrm{RP}$ is one of the most challenging clinical complications of radiotherapy for lung malignancies. At present, the molecular mechanism of its pathogenesis mainly has the following points. First, the free radicals produced by radiotherapy can be treated with amphostine. Second, recruit inflammatory cells, which can be treated with the drug celecoxib. Third, cytokines and growth factors, therapeutic drugs TGF- $\beta$ inhibitors (SM16) are still in basic research. Fourth, angiotensin-converting enzyme inhibitors (ACEI) have a potential role in reducing radiation-induced lung injury. However, the therapeutic effect is not good. The traditional Chinese medicine RSM has been widely used in the treatment of $\mathrm{RP}$ in China and many Chinese literature reports have been published, but the mechanism has not been clarified yet.

In our study, we obtained 65 effective compounds of RSM from the database and predicted 165 possible target genes, while we screened out 2,162 genes associated with radioactive 
TABLE 1 | The information on the active ingredients of Salvia miltiorrhiza.

\begin{tabular}{|c|c|c|c|}
\hline MOL ID & Molecule Name & OB & DL \\
\hline MOL001601 & 1,2,5,6-tetrahydrotanshinone & 38.75 & 0.36 \\
\hline MOL001659 & Poriferasterol & 43.83 & 0.76 \\
\hline MOL001771 & poriferast-5-en-3beta-ol & 36.91 & 0.75 \\
\hline MOL001942 & isoimperatorin & 45.46 & 0.23 \\
\hline MOL002222 & sugiol & 36.11 & 0.28 \\
\hline MOL002651 & Dehydrotanshinone II A & 43.76 & 0.4 \\
\hline MOL002776 & Baicalin & 40.12 & 0.75 \\
\hline MOL000569 & digallate & 61.85 & 0.26 \\
\hline MOL000006 & luteolin & 36.16 & 0.25 \\
\hline MOL006824 & $\alpha$-amyrin & 39.51 & 0.76 \\
\hline MOL007036 & 5,6-dihydroxy-7-isopropyl-1,1-dimethyl-2,3-dihydrophenanthren-4-one & 33.77 & 0.29 \\
\hline MOL007041 & 2-isopropyl-8-methylphenanthrene-3,4-dione & 40.86 & 0.23 \\
\hline MOL007045 & $3 \alpha$-hydroxytanshinone II a & 44.93 & 0.44 \\
\hline MOL007048 & (E)-3-[2-(3,4-dihydroxyphenyl)-7-hydroxy-benzofuran-4-yl]acrylic acid & 48.24 & 0.31 \\
\hline MOL007049 & 4-methylenemiltirone & 34.35 & 0.23 \\
\hline MOL007050 & 2-(4-hydroxy-3-methoxyphenyl)-5-(3-hydroxypropyl)-7-methoxy-3-benzofurancarboxaldehyde & 62.78 & 0.4 \\
\hline MOL007051 & 6-o-syringyl-8-o-acetyl shanzhiside methyl ester & 46.69 & 0.71 \\
\hline MOL007058 & formyltanshinone & 73.44 & 0.42 \\
\hline MOL007059 & 3-beta-Hydroxymethyllenetanshiquinone & 32.16 & 0.41 \\
\hline MOL007061 & Methylenetanshinquinone & 37.07 & 0.36 \\
\hline MOL007063 & przewalskin a & 37.11 & 0.65 \\
\hline MOL007064 & przewalskin b & 110.32 & 0.44 \\
\hline MOL007068 & Przewaquinone B & 62.24 & 0.41 \\
\hline MOL007069 & przewaquinone c & 55.74 & 0.4 \\
\hline MOL007070 & (6S,7R)-6,7-dihydroxy-1,6-dimethyl-8,9-dihydro-7H-naphtho[8,7-g]benzofuran-10,11-dione & 41.31 & 0.45 \\
\hline MOL007071 & przewaquinone $\mathrm{f}$ & 40.31 & 0.46 \\
\hline MOL007077 & sclareol & 43.67 & 0.21 \\
\hline MOL007079 & tanshinaldehyde & 52.47 & 0.45 \\
\hline MOL007081 & Danshenol B & 57.95 & 0.56 \\
\hline MOL007082 & Danshenol A & 56.97 & 0.52 \\
\hline MOL007085 & Salvilenone & 30.38 & 0.38 \\
\hline MOL007088 & cryptotanshinone & 52.34 & 0.4 \\
\hline MOL007093 & dan-shexinkum d & 38.88 & 0.55 \\
\hline MOL007094 & danshenspiroketallactone & 50.43 & 0.31 \\
\hline MOL007098 & deoxyneocryptotanshinone & 49.4 & 0.29 \\
\hline MOL007100 & dihydrotanshinlactone & 38.68 & 0.32 \\
\hline MOL007101 & dihydrotanshinone I & 45.04 & 0.36 \\
\hline MOL007105 & epidanshenspiroketallactone & 68.27 & 0.31 \\
\hline MOL007107 & C09092 & 36.07 & 0.25 \\
\hline MOL007108 & isocryptotanshi-none & 54.98 & 0.39 \\
\hline MOL007111 & Isotanshinone || & 49.92 & 0.4 \\
\hline MOL007115 & manool & 45.04 & 0.2 \\
\hline MOL007118 & microstegiol & 39.61 & 0.28 \\
\hline MOL007119 & miltionone I & 49.68 & 0.32 \\
\hline MOL007120 & miltionone II & 71.03 & 0.44 \\
\hline MOL007121 & miltipolone & 36.56 & 0.37 \\
\hline MOL007122 & Miltirone & 38.76 & 0.25 \\
\hline MOL007123 & miltirone II & 44.95 & 0.24 \\
\hline MOL007124 & neocryptotanshinone ii & 39.46 & 0.23 \\
\hline MOL007125 & neocryptotanshinone & 52.49 & 0.32 \\
\hline MOL007127 & 1-methyl-8,9-dihydro-7H-naphtho[5,6-g]benzofuran-6,10,11-trione & 34.72 & 0.37 \\
\hline MOL007130 & prolithospermic acid & 64.37 & 0.31 \\
\hline MOL007132 & (2R)-3-(3,4-dihydroxyphenyl)-2-[(Z)-3-(3,4-dihydroxyphenyl)acryloyl]oxy-propionic acid & 109.38 & 0.35 \\
\hline MOL007140 & (Z)-3-[2-[(E)-2-(3,4-dihydroxyphenyl)vinyl]-3,4-dihydroxy-phenyl]acrylic acid & 88.54 & 0.26 \\
\hline MOL007141 & salvianolic acid g & 45.56 & 0.61 \\
\hline MOL007142 & salvianolic acid j & 43.38 & 0.72 \\
\hline MOL007143 & salvilenone I & 32.43 & 0.23 \\
\hline MOL007145 & salviolone & 31.72 & 0.24 \\
\hline MOL007149 & NSC 122421 & 34.49 & 0.28 \\
\hline MOL007150 & (6S)-6-hydroxy-1-methyl-6-methylol-8,9-dihydro-7H-naphtho[8,7-g]benzofuran-10,11-quinone & 75.39 & 0.46 \\
\hline MOL007151 & Tanshindiol B & 42.67 & 0.45 \\
\hline MOL007152 & Przewaquinone $\mathrm{E}$ & 42.85 & 0.45 \\
\hline MOL007154 & tanshinone iia & 49.89 & 0.4 \\
\hline MOL007155 & (6S)-6-(hydroxymethyl)-1,6-dimethyl-8,9-dihydro-7H-naphtho[8,7-g]benzofuran-10,11-dione & 65.26 & 0.45 \\
\hline MOL007156 & tanshinone VI & 45.64 & 0.3 \\
\hline
\end{tabular}

OB, Oral Bioavailability; DL, Drug-likeness. 


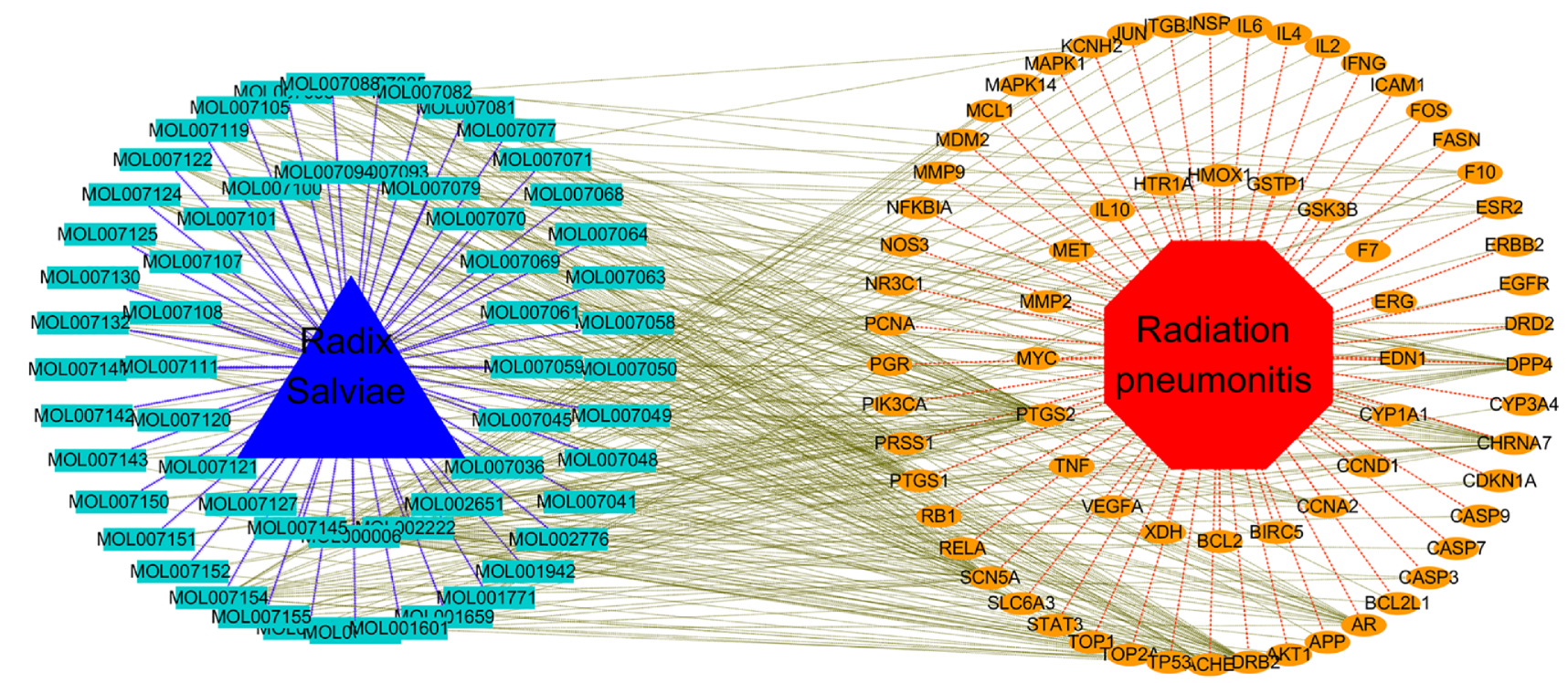

FIGURE 2 | Topological network of Drug, Chemical components, Putative target, Disease. (The red octagon represents the disease, the orange ovals represent the putative targets, the blue triangle represents the drug, and the pale blue rectangles represent the chemical components).
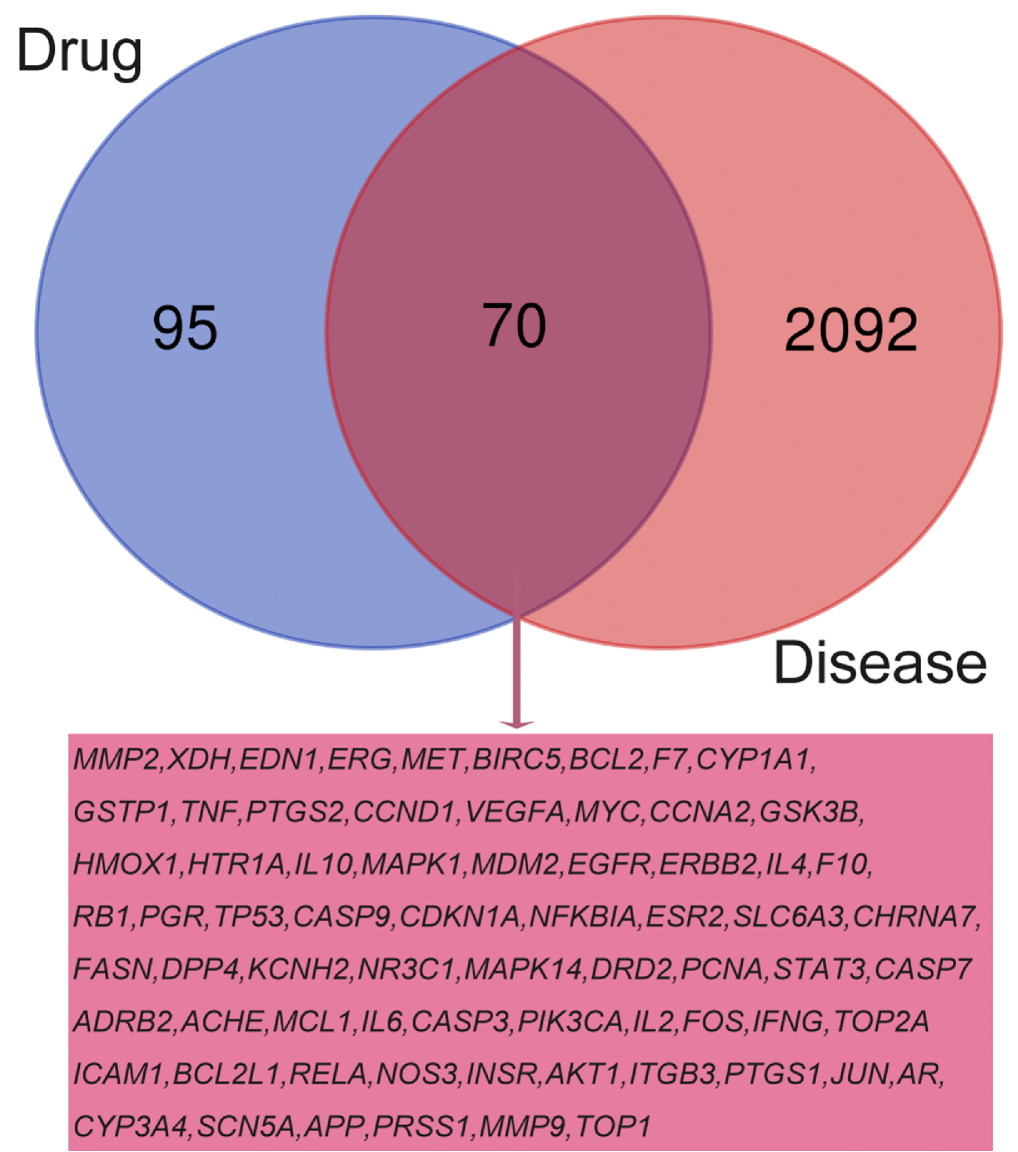

FIGURE 3 | Venn diagram for drug prediction of target genes and disease-related genes. 


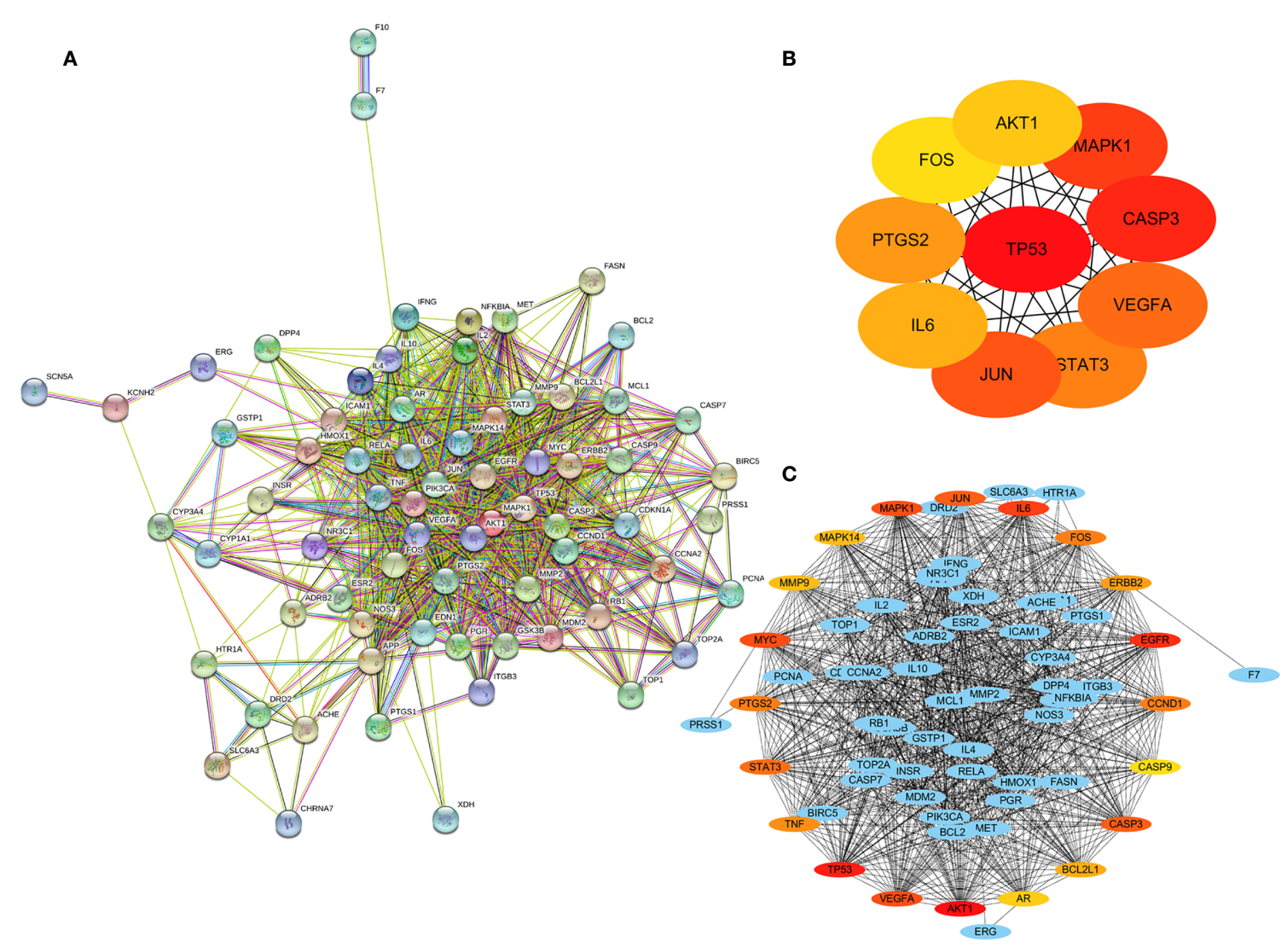

FIGURE 4 | Protein-protein interaction (PPI) network of 70 common genes. (A) PPI network of common genes, (B) Top 10 hub genes of 70 common genes by MCC algorithm. (C) Top 20 hub genes of 70 common genes with expanded subnetwork.

pneumonia. By intersections of these genes, we obtained 70 common genes, which may regulate the pharmacological effects of these genes on radiation pneumonia. By establishing the network between drugs, effective compounds, target genes, and diseases (Figure 2), we were surprised to find that 50 active components of RP act on the target gene PTGS2, while the active component MOL000006 luteolin acts on 43 target genes simultaneously.

The full name of PTGS2 is prostaglandin peroxidase 2, also known as cyclooxygenase-2 (cox-2), which is a key enzyme in prostaglandin biosynthesis and has the dual function of dioxygenase and peroxidase. The expression of PTGS2 is positively correlated with the production of ROS and inflammatory signals in tissues, and inhibiting cox- 2 can reduce inflammatory symptoms (23). The most studied cox-2 inhibitor, Celecoxib, has been shown to reduce the toxicity of radiation to the lungs (24). The possible mechanism by which this leads to radiation protection is activation of PTEN and inactivation of AKT (25). Luteolin is an active flavonoid compound with anti-oxidative, anti-inflammatory, and antifibrotic properties, which also alleviates collagen deposition, TGF- $\beta 1$ expression, and lung fibrosis (26). Luteolin acts on hepatic stellate cells to anti-fibrosis via AKT/mTOR/p70S6K and TGF $\beta /$ Smad signaling pathways (27).
PTGS2 and Luteolin are only the tip of the iceberg in the mechanism of resistance of RSM against RP, and its efficacy must be the result of multi-target and multi-pathway action. In order to further reveal the mechanism, we uploaded the obtained 70 common genes to the STRING website to obtain their PPI network, and we obtained the hub genes through two algorithms (MMC and Degree), which were displayed in multiple ways (Figure 4).

Activation of the STAT3 pathway may play an important role in the pathogenesis of radiation lung injury. The protective effect of delayed treatment of WP1066 suggests that the STAT3 signal may be a therapeutic target for RP (28). Clarithromycin can prevent radiation pneumonia by PTGS2, TNF-antigen, TNF receptor 1, NF-B, vcam-1, and MMP9 (29). Interleukin 6 (IL6) has been reported as a risk factor for RP and contributes to the development of RP (30). FOS plays an important role in the radiation resistance mechanism of malignant glioma and may be a potential new target for the treatment of malignant glioma (31). Many of the above genes play an important role in radiation resistance, some have been reported in RP, and some have not been published yet, which suggests that we should carry out relevant research and could find potential key genes related to RP in them. 
A

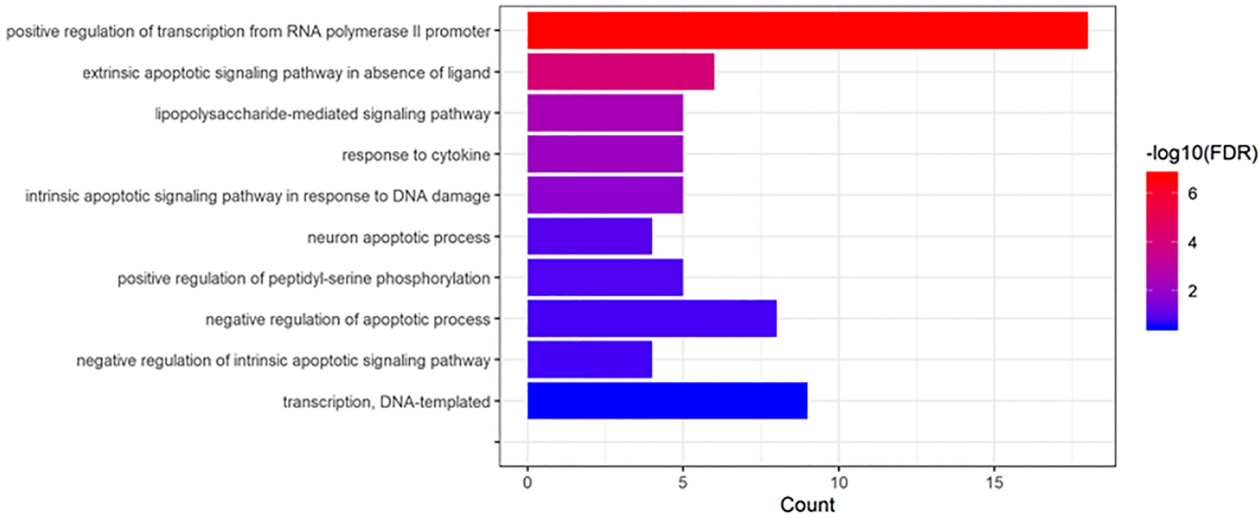

B

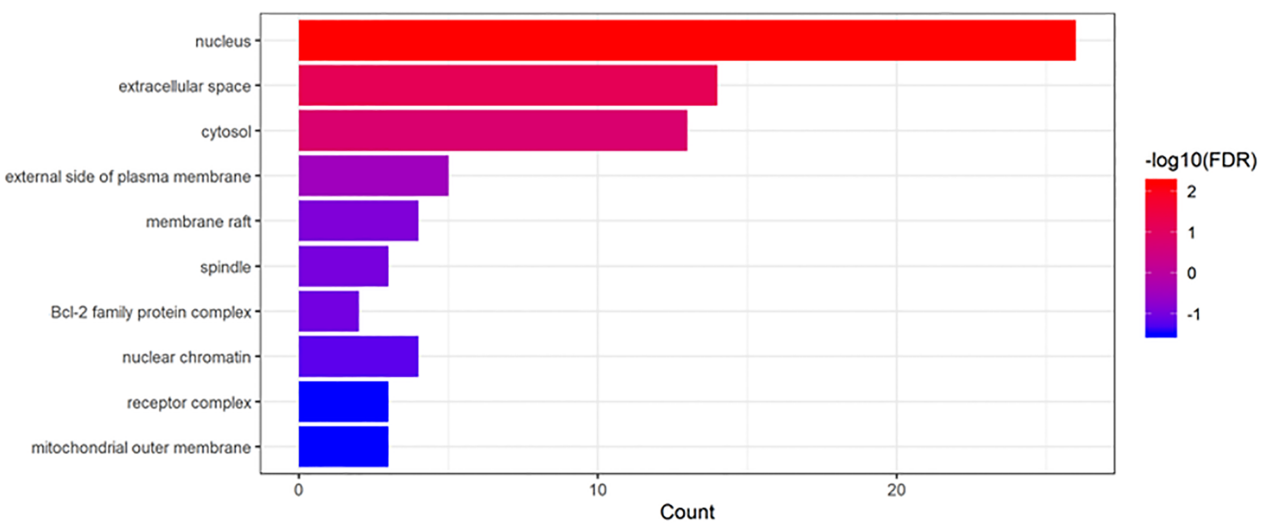

C

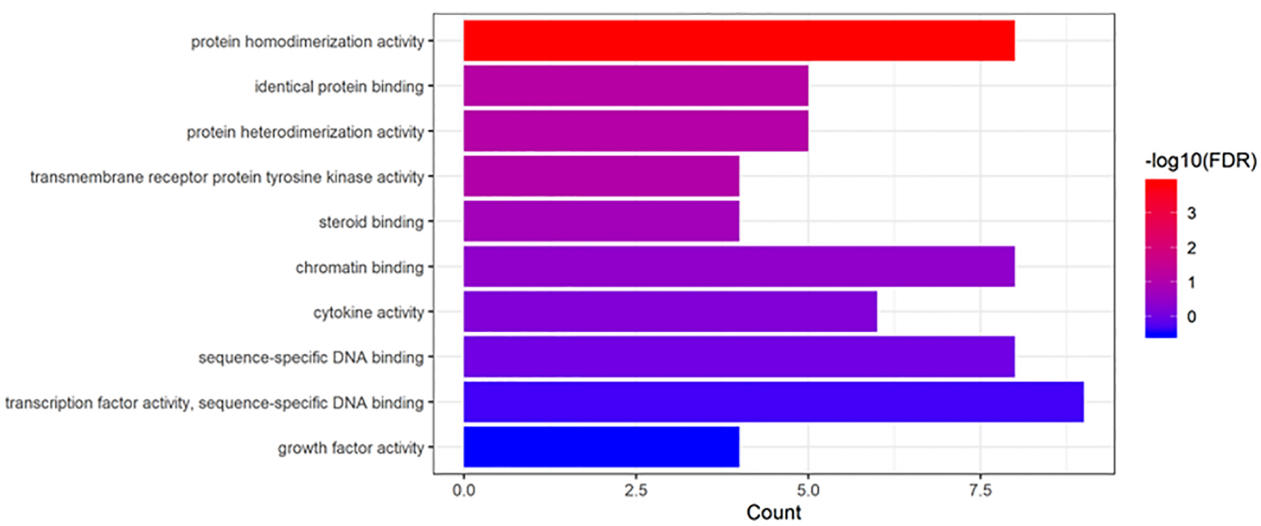

FIGURE 5 | Gene Ontology enrichment analysis of common genes. (A) Biological process, (B) cellular component, (C) molecular function.

In addition, to further understand the interaction between these common genes, we enriched their GO function and analyzed their KEGG pathway. Through our research, we found that RSM may regulate protein homodimerization activity via positive regulation of transcription from RNA polymerase II promoter, extrinsic apoptotic signaling pathway in the absence of ligand- and lipopolysaccharide-mediated signaling pathway, and response to cytokine in the nucleus to anti-radiation pneumonia. Many proteins need to function as homologous dimers; affecting the activity of protein homologous dimer will lead to the loss of its function (32). The repair mechanism of DNA double-strand break includes homologous recombination and non-homologous end joining (33). The change of protein homodimerization activity must lead to the resistance or sensitivity of cells to radiation. Although not experimentally confirmed, there is reason to believe that RSM may improve the body's ability to repair radiation damage through homologous recombination. 


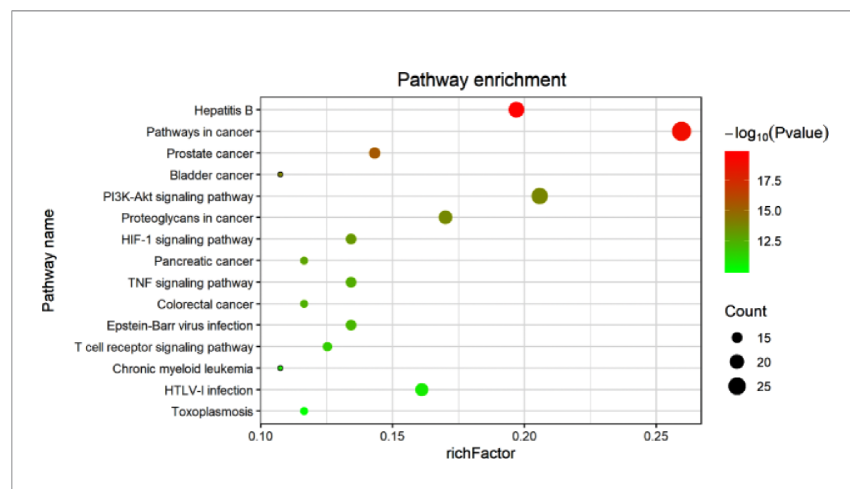

FIGURE 6 | KEGG pathway analysis for common genes.

Through KEGG analysis, multiple signaling pathways may be the underlying mechanism. Figure 7 shows the three pathways and highlights some of the 70 genes in common. PI3K-AKT, HIF, and TNF signaling pathways have all been reported in radiation pneumonia. Tang $\mathrm{Y}$ et al. reported that severe radiation pneumonia was associated with genetic variation in the PI3K/ AKT pathway in patients with lung cancer after radiotherapy (34). Study of Toullec, A et al. has shown that loss of HIF in intestinal endothelial cells can reduce the severity of radiation enteritis, but similar loss of intestinal epithelial cells cannot (35). Loss of HIF-1 $\alpha$ does not have a beneficial effect on lung injury in mice during stereotactic radiotherapy (36). Zhang, $M$ et al. demonstrated the selective protective effect of TNF- $\alpha$ pathway inhibition on radiation lung injury by gene knockout and antisense oligonucleotide (ASO) silencing of TNF- $\alpha$ in mice model of lung metastasis of colon cancer (37). Multiple target genes of RSM are enriched in the three pathways mentioned above, and the roles of these pathways are mainly focused on cell survival, cell cycle progression, cell proliferation, angiogenesis, DNA repair, reducing oxygen consumption, regulating proliferation and apoptosis, and synthesis of inflammatory mediators. The complexity of its functions exactly reflects the multi-component, multi-target, and multi-pathway pharmacological mechanism of RSM. The above analysis points out the direction of our further research.

Our study had several limitations. First of all, more Chinese medicine target gene databases and more comprehensive disease prediction databases are needed to make the combined analysis results more reliable. The second is that generic databases and analytics alone are not enough to clarify the actual mechanism. Our results need to be further validated in cell and animal models. The comprehensive understanding of RSM and RP depends on the common development of multi-disciplines.

\section{CONCLUSION}

By means of network pharmacology, our study predicted the effective components of RSM and explored the underlying mechanism of the potential anti-RP effect most likely to focus on luteolin and be used as a PTSG2 target. Through the

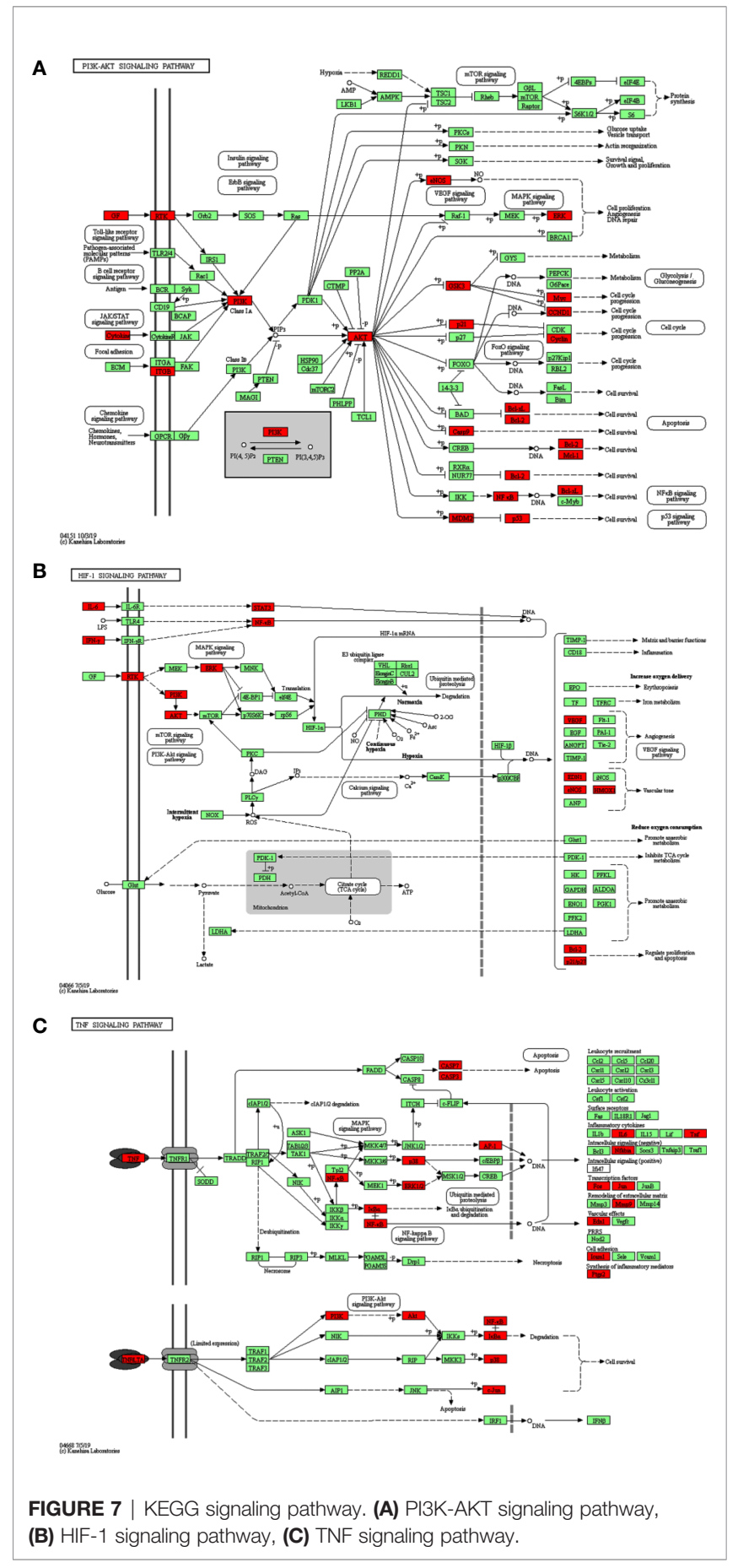

analysis of specific signaling pathways, we believe that RSM may pass through the main PI3K-AKT, HIF-1, and TNF signaling pathways. The mechanism of resistance to radiation pneumonia is the direct or indirect synergistic effect of multiple targets and multiple pathways, rather than the result of single target and single pathway. This study revealed the potential mechanism of RSM resistance to radiation pneumonia in theory, but further experimental verification is needed to clarify its true internal mechanism. 


\section{DATA AVAILABILITY STATEMENT}

The original contributions presented in the study are included in the article/Supplementary Material. Further inquiries can be directed to the corresponding authors.

\section{AUTHOR CONTRIBUTIONS}

PL completed most of the research and drafted manuscripts. XX plotted all of the charts. JZ analyzed the data. JW designed the research and reviewed the manuscript. All authors contributed to the article and approved the submitted version.

\section{REFERENCES}

1. Lu L, Sun C, Su Q, Wang Y, Li J, Guo Z, et al. Radiation-Induced Lung Injury: Latest Molecular Developments, Therapeutic Approaches, and Clinical Guidance. J Thorac Oncol (2019) 19(4):417-26. doi: 10.1007/s10238-019-00571-w

2. Madani I, De Ruyck K, Goeminne H, De Neve W, Thierens H, Van Meerbeeck J. Predicting Risk of Radiation-Induced Lung Injury. J Thorac Oncol (2007) 2(9):864-74. doi: 10.1097/JTO.0b013e318145b2c6

3. Williams JP, Johnston CJ, Finkelstein JN. Treatment for Radiation-Induced Pulmonary Late Effects: Spoiled for Choice or Looking in the Wrong Direction? Curr Drug Targets (2010) 11(11):1386-94. doi: 10.2174/ 1389450111009011386

4. Qi F, Zhao L, Zhou A, Zhang B, Li A, Wang Z, et al. The Advantages of Using Traditional Chinese Medicine as an Adjunctive Therapy in the Whole Course of Cancer Treatment Instead of Only Terminal Stage of Cancer. Biosci Trends (2015) 9(1):16-34. doi: 10.5582/bst.2015.01019

5. Pang H, Wu L, Tang Y, Zhou G, Qu C, Duan JA. Chemical Analysis of the Herbal Medicine Salviae Miltiorrhizae Radix Et Rhizoma (Danshen). Molecules (2016) 21(1):51. doi: 10.3390/molecules21010051

6. Luo Y, Feng Y, Song L, He GQ, Li S, Bai SS, et al. A Network PharmacologyBased Study on the Anti-Hepatoma Effect of Radix Salviae Miltiorrhizae. Chin Med (2019) 14:27. doi: 10.1186/s13020-019-0249-6

7. Gao LN, Cui YL, Wang QS, Wang SX. Amelioration of Danhong Injection on the Lipopolysaccharide-Stimulated Systemic Acute Inflammatory Reaction Via Multi-Target Strategy. J Ethnopharmacol (2013) 149(3):772-82. doi: 10.1016/j.jep.2013.07.039

8. Wang L, Ma R, Liu C, Liu H, Zhu R, Guo S, et al. Salvia Miltiorrhiza: A Potential Red Light to the Development of Cardiovascular Diseases. Curr Pharm Des (2017) 23(7):1077-97. doi: 10.2174/1381612822666161010105242

9. XD ME, Cao YF, Che YY, Li J, Shang ZP, Zhao WJ, et al. Danshen: A Phytochemical and Pharmacological Overview. Chin J Nat Med (2019) 17 (1):59-80. doi: 10.1016/S1875-5364(19)30010-X

10. Li QL, Liu JL, Wang JR, Jian XD, Kan BT, Zhang ZC. The Study of Using Cordyceps-Astragalus-Salvia Miltiorrhiza to Treat Acute Lung Injury Induced by Paraquat Poisoning in Rats. Zhonghua Lao Dong Wei Sheng Zhi Ye Bing Za Zhi (2017) 35(4):251-6. doi: 10.3760/cma.j.issn.1001-9391.2017.04.003

11. Liu MW, Wang YH, Qian CY, Li H. Xuebijing Exerts Protective Effects on Lung Permeability Leakage and Lung Injury by Upregulating Toll-Interacting Protein Expression in Rats With Sepsis. Int J Mol Med (2014) 34(6):1492-504. doi: 10.3892/ijmm.2014.1943

12. Wan LM, Tan L, Wang ZR, Liu SX, Wang YL, Liang SY, et al. Preventive and Therapeutic Effects of Danhong Injection on Lipopolysaccharide Induced Acute Lung Injury in Mice. J Ethnopharmacol (2013) 149(1):352-9. doi: 10.1016/j.jep.2013.06.048

13. Zhang M, Cao SR, Zhang R, Jin JL, Zhu YF. The Inhibitory Effect of Salvianolic Acid B on TGF-betal-Induced Proliferation and Differentiation in Lung Fibroblasts. Exp Lung Res (2014) 40(4):172-85. doi: 10.3109/ 01902148.2014.895070

14. Du S, Yao Q, Tan P, Xie G, Ren C, Sun Q, et al. Protective Effect of Tanshinone IIA Against Radiation-Induced Ototoxicity in HEI-OC1 Cells. Oncol Lett (2013) 6(4):901-6. doi: 10.3892/ol.2013.1486

\section{FUNDING}

This work was supported by the National Natural Science Foundation of China (81672975), the Six Talent Peaks Project of Jiangsu Province of China (WSN095).

\section{SUPPLEMENTARY MATERIAL}

The Supplementary Material for this article can be found online at: https://www.frontiersin.org/articles/10.3389/fonc.2021.684315/ full\#supplementary-material

15. Cao HY, Ding RL, Li M, Yang MN, Yang LL, Wu JB, et al. Danshensu, A Major Water-Soluble Component of Salvia miltiorrhiza, Enhances the Radioresponse for Lewis Lung Carcinoma Xenografts in Mice. Oncol Lett (2017) 13(2):605-12. doi: 10.3892/ol.2016.5508

16. Hopkins AL. Network Pharmacology: The Next Paradigm in Drug Discovery. Nat Chem Biol (2008) 4(11):682-90. doi: 10.1038/nchembio.118

17. Li S, Zhang B. Traditional Chinese Medicine Network Pharmacology: Theory, Methodology and Application. Chin J Nat Med (2013) 11(2):110-20. doi: 10.1016/S1875-5364(13)60037-0

18. Ru J, Li P, Wang J, Zhou W, Li B, Huang C, et al. TCMSP: A Database of Systems Pharmacology for Drug Discovery From Herbal Medicines. J Cheminform (2014) 6:13. doi: 10.1186/1758-2946-6-13

19. Safran M, Chalifa-Caspi V, Shmueli O, Olender T, Lapidot M, Rosen N, et al. Human Gene-Centric Databases at the Weizmann Institute of Science: Genecards, UDB, Crow 21 and HORDE. Nucleic Acids Res (2003) 31 (1):142-6. doi: 10.1093/nar/gkg050

20. Szklarczyk D, Franceschini A, Wyder S, Forslund K, Heller D, Huerta-Cepas J, et al. String v10: Protein-Protein Interaction Networks, Integrated Over the Tree of Life. Nucleic Acids Res (2015) 43(Database issue):D447-52. doi: $10.1093 /$ nar/gku1003

21. Huang da W, Sherman BT, Lempicki RA. Systematic and Integrative Analysis of Large Gene Lists Using DAVID Bioinformatics Resources. Nat Protoc (2009) 4(1):44-57. doi: 10.1038/nprot.2008.211

22. Huang da W, Sherman BT, Lempicki RA. Bioinformatics Enrichment Tools: Paths Toward the Comprehensive Functional Analysis of Large Gene Lists. Nucleic Acids Res (2009) 37(1):1-13. doi: 10.1093/nar/gkn923

23. Cheki M, Yahyapour R, Farhood B, Rezaeyan A, Shabeeb D, Amini P, et al. COX-2 in Radiotherapy: A Potential Target for Radioprotection and Radiosensitization. Curr Mol Pharmacol (2018) 11(3):173-83. doi: 10.2174/ 1874467211666180219102520

24. Hunter NR, Valdecanas D, Liao Z, Milas L, Thames HD, Mason KA. Mitigation and Treatment of Radiation-Induced Thoracic Injury With a Cyclooxygenase-2 Inhibitor, Celecoxib. Int J Radiat Oncol Biol Phys (2013) 85(2):472-6. doi: 10.1016/j.ijrobp.2012.04.025

25. Meng Z, Gan YH. Activating PTEN by COX-2 Inhibitors Antagonizes RadiationInduced AKT Activation Contributing to Radiosensitization. Biochem Biophys Res Commun (2015) 460(2):198-204. doi: 10.1016/j.bbrc.2015.03.008

26. Chen CY, Peng WH, Wu LC, Wu CC, Hsu SL. Luteolin Ameliorates Experimental Lung Fibrosis Both In Vivo and In Vitro: Implications for Therapy of Lung Fibrosis. J Agric Food Chem (2010) 58(22):11653-61. doi: $10.1021 /$ jf1031668

27. Li J, Li X, Xu W, Wang S, Hu Z, Zhang Q, et al. Antifibrotic Effects of Luteolin on Hepatic Stellate Cells and Liver Fibrosis by Targeting AKT/mTOR/p70S6K and TGFbeta/Smad Signalling Pathways. Liver Int (2015) 35(4):1222-33. doi: 10.1111/liv.12638

28. Yu J, Yuan X, Liu Y, Zhang K, Wang J, Zhang H, et al. Delayed Administration of WP1066, an STAT3 Inhibitor, Ameliorates Radiation-Induced Lung Injury in Mice. Lung (2016) 194(1):67-74. doi: 10.1007/s00408-015-9821-8

29. Lee SJ, Yi CO, Heo RW, Song DH, Cho YJ, Jeong YY, et al. Clarithromycin Attenuates Radiation-Induced Lung Injury in Mice. PloS One (2015) 10(6): e0131671. doi: 10.1371/journal.pone.0131671 
30. Fu ZZ, Peng Y, Cao LY, Chen YS, Li K, Fu BH. Correlations Between Serum IL-6 Levels and Radiation Pneumonitis in Lung Cancer Patients: A MetaAnalysis. J Clin Lab Anal (2016) 30(2):145-54. doi: 10.1002/jcla.21828

31. Liu ZG, Jiang G, Tang J, Wang H, Feng G, Chen F, et al. c-Fos OverExpression Promotes Radioresistance and Predicts Poor Prognosis in Malignant Glioma. Oncotarget (2016) 7(40):65946-56. doi: 10.18632/ oncotarget.11779

32. Li Z, Peng H, Qin L, Qi J, Zuo X, Liu JY, et al. Determinants of 14-3-3sigma Protein Dimerization and Function in Drug and Radiation Resistance. J Biol Chem (2013) 288(44):31447-57. doi: 10.1074/jbc.M113.467753

33. Rastogi RP, Richa, Kumar A, Tyagi MB, Sinha RP. Molecular Mechanisms of Ultraviolet Radiation-Induced DNA Damage and Repair. J Nucleic Acids (2010) 2010:592980. doi: 10.4061/2010/592980

34. Tang Y, Liu B, Li J, Wu H, Yang J, Zhou X, et al. Genetic Variants in PI3K/ AKT Pathway Are Associated With Severe Radiation Pneumonitis in Lung Cancer Patients Treated With Radiation Therapy. Cancer Med (2016) 5 (1):24-32. doi: 10.1002/cam4.564

35. Toullec A, Buard V, Rannou E, Tarlet G, Guipaud O, Robine S, et al. HIF1alpha Deletion in the Endothelium, But Not in the Epithelium, Protects From Radiation-Induced Enteritis. Cell Mol Gastroenterol Hepatol (2018) 5 (1):15-30. doi: 10.1016/j.jcmgh.2017.08.001

36. Lavigne J, Suissa A, Verger N, Dos Santos M, Benadjaoud M, Mille-Hamard L, et al. Lung Stereotactic Arc Therapy in Mice: Development of Radiation
Pneumopathy and Influence of HIF-1alpha Endothelial Deletion. Int J Radiat Oncol Biol Phys (2019) 104(2):279-90. doi: 10.1016/j.ijrobp.2019.01.081

37. Zhang M, Qian J, Xing X, Kong FM, Zhao L, Chen M, et al. Inhibition of the Tumor Necrosis Factor-Alpha Pathway Is Radioprotective for the Lung. Clin Cancer Res (2008) 14(6):1868-76. doi: 10.1158/1078-0432.CCR-07-1894

Conflict of Interest: The authors declare that the research was conducted in the absence of any commercial or financial relationships that could be construed as a potential conflict of interest.

Publisher's Note: All claims expressed in this article are solely those of the authors and do not necessarily represent those of their affiliated organizations, or those of the publisher, the editors and the reviewers. Any product that may be evaluated in this article, or claim that may be made by its manufacturer, is not guaranteed or endorsed by the publisher.

Copyright (c) $2021 \mathrm{Li}, \mathrm{Xia}$, Zhou and Wu. This is an open-access article distributed under the terms of the Creative Commons Attribution License (CC BY). The use, distribution or reproduction in other forums is permitted, provided the original author(s) and the copyright owner(s) are credited and that the original publication in this journal is cited, in accordance with accepted academic practice. No use, distribution or reproduction is permitted which does not comply with these terms. 